\title{
Impactos dendrométricos e econômicos de um povoamento de Pinus elliottii submetidos a produção de resina
}

\author{
Bruno de Almeida Lima' ${ }^{1}$, Marcos Felipe Nicoletti2 ${ }^{2}$, Thiago Floriani Stepka ${ }^{2}$, Philipe Ricardo \\ Casemiro Soares ${ }^{2}$, Maycon Thuan Saturnino Da Silva ${ }^{3}$
}

\author{
${ }^{1}$ Universidade Estadual do Centro-Oeste, Rua Professora Maria Roza Zanon de Almeida Engenheiro, Gutierrez, CEP: 84505-677, Irati - PR, \\ Brasil \\ ${ }^{2}$ Universidade do Estado de Santa Catarina, Av. Luiz de Camões, 2090, Conta Dinheiro, CEP: 88.520-000, Lages /-SC, Brasil \\ ${ }^{3}$ Universidade Federal do Paraná, Av. Pref. Lothário Meissner, 632, Jardim Botânico, Campus III, CEP 80210-170, Curitiba, PR, Brasil
}

\begin{abstract}
Original Article
*Corresponding author: bruno.crvg22@gmail.com

Keywords:

Economic analysis

Forest growth

Tapper

Palavras-chave:

Análise econômica

Crescimento florestal

Afilamento

Received in

2019/12/19

RESUMO: O trabalho tem como objetivo verificar a viabilidade econômica da técnica de resinagem e caracterização dendrométricas do diâmetro, altura e volume em povoamento de Pinus elliottii Eng. Var elliottii para duas densidades populacionais. Os dados foram coletados num povoamento de Pinus elliottii Eng. Var elliottii com área total de 4 hectares, situado no município de Balneário Pinhal, no estado do Rio Grande do Sul. Os 4 hectares foram divididos em duas áreas igualitárias, de acordo com a densidade populacional e as intervenções silviculturais. Foram selecionadas 30 árvores para cada área. Coletou-se seis discos por árvore, nas alturas absolutas de 0,1 e 1,3 m e nas alturas relativas de $25 \%, 50 \%, 75 \%$ da altura total da árvore (ht) e na altura comercial (diâmetro igual a $8 \mathrm{~cm}$ ). Para a modelagem de crescimento da área 1 e 2 das variáveis dendrométricas (diâmetro, altura e volume) foi utilizado o modelo de Chapman Richards. Para estimar o volume e diâmetro de secção foi usado o modelo de afilamento Schöepfer. Na análise econômica foram determinados 6 cenários e utilizados os métodos VPL, TIR e Razão B/C. Há uma queda na curva de crescimento das árvores resinadas da área $1 \mathrm{e} 2$ a partir da idade 11 . O processo de resinagem provocou redução nas variáveis dendrométricas em comparação com árvores não resinadas. Os cenários com técnica de resinagem obtiveram lucros superiors.
\end{abstract}

Accepted on

2021/05/04

Published in

2021/08/31

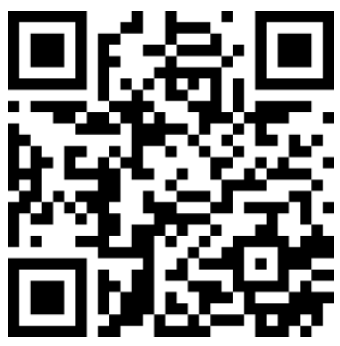

DOI: http://dx.doi.org/ 10.34062/afs.v8i2.9357

\section{(cc) BY}

Adv. For. Sci, Cuiabá, v. 8, n. 2, p. 1475-1487, 2021

\section{Dendrometric and economic impacts of a Pinus elliottii stand used in resin production}

\begin{abstract}
The objective of this work is to verify the economic viability of the resin technique and dendrometric characterization of Pinus elliottii Eng. var elliottii stand diameter, height, and volume for two population densities. Data were collected from a Pinus elliottii Eng. Var elliottii stand with a total area of 4 hectares, located in the municipality of Balneário Pinhal, in the state of Rio Grande do Sul. The 4 hectares were divided into two equal areas, according to the density. population and forestry interventions. The complete trunk analysis technique (ANATRO) was used for growth reconstruction and future projections on 30 representative trees of each area. For this, six disks per tree were collected, at absolute heights of 0.1 and $1.3 \mathrm{~m}$ and at relative heights of $25 \%, 50 \%, 75 \%$ of total tree height (ht) and $100 \%$ at commercial height. (diameter equal to $8 \mathrm{~cm}$ ). For the growth modelling of area 1 and 2 of the dendrometric variables (diameter, height, and volume) the Chapman - Richards model was used. To estimate section, volume, and diameter, the Schöepfertaper model was used. For economic analysis, 6 scenarios were determined and the NPV, IRR and B/C ratio methods were used. There is a decrease in the growth curve of area 1 and 2 resin trees from age 11. The resin process reduced dendrometric variables compared to non-resin trees. The scenarios with resin toxique obtained superior profits.
\end{abstract}


Introdução

As áreas das florestas plantadas no Brasil totalizam 7,84 hectares e desse total os plantios de Pinus sp. ocupam 1,58 hectares, concentrados principalmente no estado de Santa Catarina com $36 \%$ e tem destino em grande parte para o setor de papel e celulose, madeira serrada. Esse gênero se adapta ao sul do país pelas melhores condições de solo e clima (IBÁ, 2019). Além dos produtos derivados citados anteriormente as espécies do gênero Pinus são as principais produtoras de resina, que é uma classe de substância que se encontra nas árvores e é extraído. O Pinus tem uma boa aptidão e viabilidade para produção de resina devido aos canais de resina serem formados por células epiteliais com paredes fina, e a prática da resinagem aumenta a frequência desses canais por consequência aumenta a produção de resina. (De Cademartori et al. 2012; Ferreira 2014).

A resinagem no Brasil teve início a partir de 1970 com o estabelecimento dos grandes maciços florestais de Pinus elliottii plantados com incentivos governamentais (Garrido et al.1998). Desde então, a resinagem teve grandes avanços, no sentido físico e químico onde influenciou no processamento de separação e purificação de resina-bruta em produto de alto valo agregado, como também na coleta do produto e aumento da produtividade por árvore a custos menores (Ferreira 2001). Nesse sentido, diversas pesquisas foram realizadas e contribuíram decisivamente para que o Brasil passasse de importador para exportador da goma resina em um curto período de tempo (Rocha 2012). Atualmente, o Brasil é o segundo maior produtor mundial de resina, perdendo apenas para China (ARESB 2017).

$\mathrm{O}$ processo de resinagem no Brasil pode ter sua importância avaliada pelo volume de produção, facilidade de industrialização da resina bruta e pela diversidade de aplicações dos produtos obtidos a partir da primeira transformação (Ferreira 2001). A resinagem pode vir a construir um ativo da economia, pois além de antecipar receitas em um povoamento florestal antes do produto (madeira), a resinagem é responsável por gerar diversos empregos, sejam eles diretos, já que todo o processo de extração da resina é manual, ou indiretos no beneficiamento da resina nas indústrias (Rodrigues 2008). A atividade de resinagem segundo Ferreira (2001), pode ser mais rentável, pois gera um aumento no total das receitas de 10 a $20 \%$ a favor da resinagem.

No entanto, Figueiredo Filho et al. (1992) apontou inconvenientes dessa técnica. Em pesquisa realizada, os autores compararam o crescimento em diâmetro, altura e volume entre árvores resinadas e não resinadas de Pinus elliottii e constataram que a resinagem influenciou na diminuição do crescimento da madeira de $14,99 \%$ em volume, $12,02 \%$ em altura e de $6 \%$ até $14,49 \%$ em diâmetro ao longo do tronco no final da rotação. Os autores também afirmam que além da diminuição do acréscimo lenhoso, o estabelecimento do painel de resinagem conduz a desclassificação da madeira da primeira tora quanto ao seu destino final, ou seja, esse material lenhoso terá um uso menos nobre, com consequente menor valor econômico no mercado consumidor.

Por meio de pesquisas, como de Lima et al. (2013), sabe-se que a densidade de árvores por hectare interfere diretamente no desenvolvimento em diâmetro e tamanho de copa, que são fatores importantes para produção de goma resina. Neves et al. (2006) estudaram a influência do diâmetro na produção de goma resina e concluiu que cada $1 \mathrm{~cm}$ de acréscimo no DAP correspondeu um aumento de 10,54 gramas de produção de goma resina por planta e por estria.

Portanto, diante disso, o presente trabalho teve por objetivo verificar a viabilidade econômica da técnica de resinagem e caracterizar as variáveis dendrométricas (diâmetro, altura e volume) do povoamento de Pinus elliottii Eng. var elliottii para duas densidades populacionais, no estado do Rio Grande do Sul.

\section{Material e Métodos}

Característica da área

A pesquisa foi realizada na área florestal da Empresa Celulose Irani S. A., situada no município de Balneário Pinhal, no estado do Rio Grande do Sul, sob as coordenadas geográficas de $50^{\circ} 22^{\prime} 55.44^{\prime \prime}$ de longitude Oeste e de $30^{\circ} 11^{\prime} 31.72$ " de latitude Sul (Figura 1).

A região tem clima do tipo $\mathrm{Cfa}$, segundo a classificação de Köeppen, com temperatura média anual de $19^{\circ} \mathrm{C}$, o índice pluviométrico anual é de $1300 \mathrm{~mm}$ (Alvares et al. 2013). O tipo de solo é Planossolo Hidromórfico eutrófico solódico (Streck 2002). 


\section{Lima et al.}
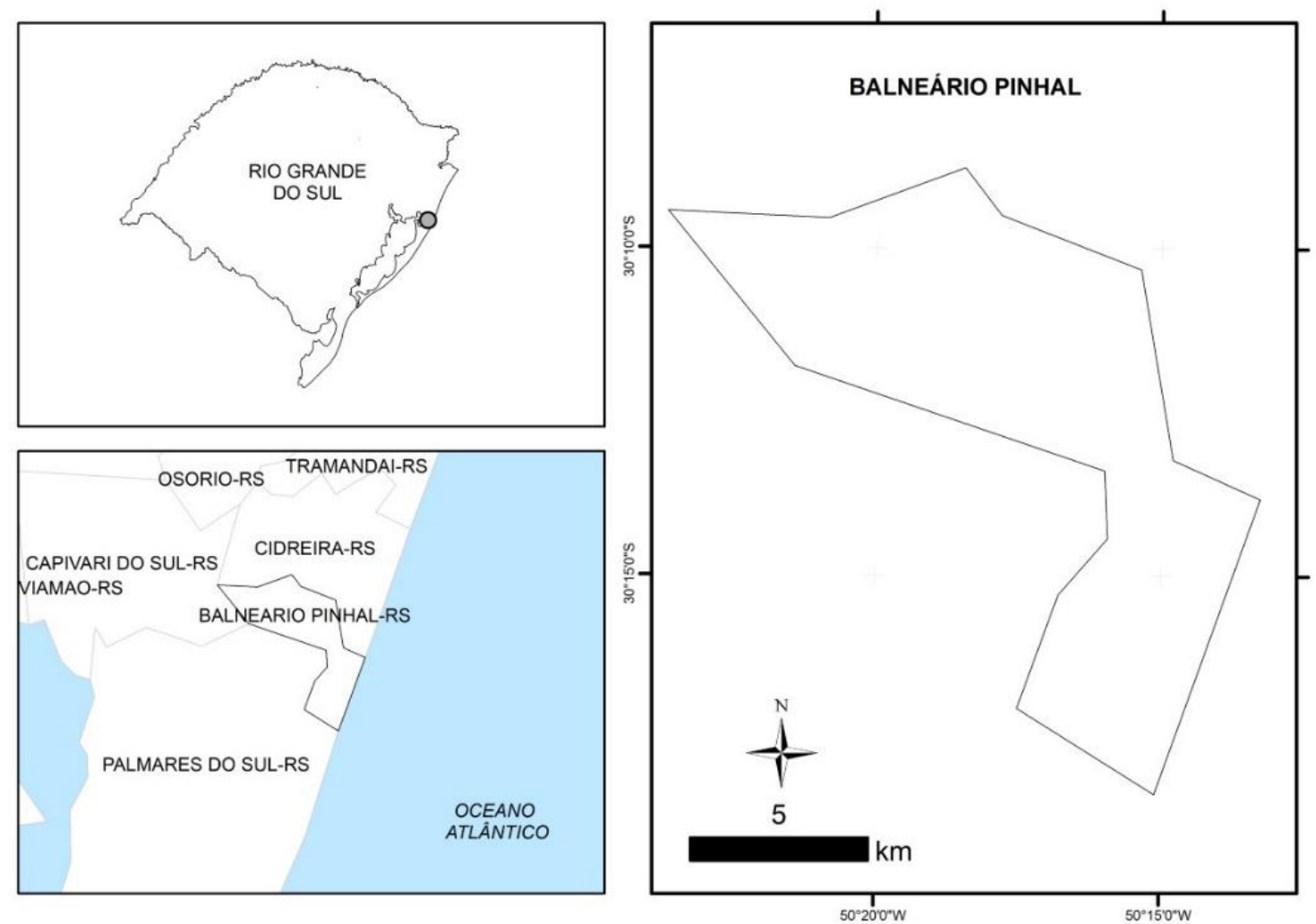

Figura 1. Localização do plantio de Pinus elliottii Eng. var elliottii no munícipio de Balneário Pinhal, Rio Grande do Sul.

\section{Levantamento de dados}

Os dados utilizados para o presente estudo foram coletados em um povoamento de Pinus elliottii Eng. var elliottii com área total de 4 hectares, que se encontrava em regeneração natural desde 2001, possuindo aproximadamente 16 anos de idade, quando realizado o estudo. Os 4 hectares do povoamento foram divididos igualmente, com dois hectares cada, sendo atribuídos os nomes "Área 1" e “Área 2", de acordo com a densidade populacional e os tratos silviculturais aplicados, conforme mostrado na Tabela 1.

Tabela 1. Caracterização do povoamento Pinus elliottii Eng. var elliottii nas áreas 1 e 2.

\begin{tabular}{lcc}
\hline Características & Área 1 & Área 2 \\
\hline Roçada & Ano 4-5 & Ano 4 \\
Desbaste & Ano 10 & Ano 4 \\
$\mathrm{~N}^{\circ}$ de árvores/ha & 800 & 1180 \\
\hline
\end{tabular}

Em ambos os cenários, as árvores remanescentes do desbaste foram escolhidas pelo maior DAP (diâmetro à altura do peito medido a 1,30 $\mathrm{m}$ do nível do solo) e pelo desenvolvimento mais uniforme do tronco.

A caracterização do povoamento foi realizada por meio do censo florestal para a Área 2, em que para todos os indivíduos foram medidos os daps e as alturas. Para Área 1, foram alocadas 8 unidades amostrais com área de $400 \mathrm{~m}^{2}(20 \times 20 \mathrm{~m})$, pelo método de área fixa. Os dados das variáveis dendrométricas foram coletados por meio do processo de amostragem aleatória simples, sendo amostrados o DAP de todas as árvores dentro de cada parcela e a altura de $20 \%$ dos indivíduos. As demais alturas foram estimadas por relação hipsométrica cuja equação foi: $h=16,1934+0,1433 *$ DAP.
Para ambas as situações, utilizou-se a suta para medição dos diâmetros (DAP) e o hipsômetro Vertex IV para medição das alturas totais (h). As razões para aplicar amostragem na área 1 e não o censo foram por causa de questões de operacionais, disponibilidade de mão-de-obra e menor tempo que a empresa exigiu visando atingir o resultado mais rápido. Por isso tem-se duas áreas com diferentes inventários quanto a obtenção de dados.

Os dados dendrométricos levantados, para ambas as áreas inventariadas, serviram como base para definir quais indivíduos seriam selecionados para o corte, visando reconstruir seu crescimento pela técnica de análise de tronco completa (ANATRO). Essa seleção das árvores foi realizada com base na distribuição diamétrica do povoamento, conforme a fórmula de Sturges (1). 
$\mathrm{NC}=1+3,3 \log n$

Em que: $\mathrm{NC}=$ Número de classes $; \mathrm{n}=$ Número de árvores medidas/amostradas.

De acordo com as classes de diâmetro encontradas para cada situação, foi possível identificar os indivíduos que seriam amostrados. Conforme o Teorema do Limite Central (PÓ, 2012), decidiu-se selecionar 30 árvores para cada área, para que se obtivesse um menor erro amostral e a distribuição pudesse chegar mais próximo da normalidade. As árvores foram coletadas de forma a atender uma homogeneidade nas classes diamétricas encontradas. Construiu-se 6 classes diamétricas nas duas áreas como mostra a tabela 2. As árvores foram coletadas de forma a atender uma homogeneidade nas classes diamétricas encontradas e uma representação fidedigna da floresta.

Tabela 2. Distribuição do número de árvore resinadas e não resinadas em 6 classes diamétricas.

\begin{tabular}{|c|c|c|c|c|c|c|c|c|}
\hline \multirow{2}{*}{$\mathbf{N}^{\circ}$ classe } & \multicolumn{4}{|c|}{ Árvores não resinadas } & \multicolumn{4}{|c|}{ Árvores resinadas } \\
\hline & $\mathbf{L i}$ & Ls & $\mathbf{N}$ & $\mathbf{n}^{\circ} \mathbf{a}$ & $\mathbf{L i}$ & Ls & $\mathbf{N}$ & $\mathbf{n}^{\circ} \mathbf{a}$ \\
\hline 1 & 16,6 & 20,6 & 47 & 5 & 16,0 & 19,0 & 6 & 2 \\
\hline 2 & 20,6 & 24,6 & 112 & 9 & 19,0 & 22,0 & 40 & 5 \\
\hline 3 & 24,6 & 28,6 & 103 & 6 & 22,0 & 25,0 & 79 & 7 \\
\hline 4 & 28,6 & 32,6 & 52 & 6 & 25,0 & 28,0 & 99 & 10 \\
\hline 5 & 32,6 & 36,6 & 9 & 2 & 28,0 & 31,0 & 22 & 4 \\
\hline 6 & 36,6 & 40,6 & 2 & 2 & 31,1 & 34,1 & 5 & 2 \\
\hline \multicolumn{4}{|c|}{ Total } & 30 & & otal & & 30 \\
\hline
\end{tabular}

$\mathrm{Li}=$ Limite inferior; $\mathrm{Ls}=$ limite superior; $\mathrm{N}=$ número de árvores, oriundas do inventário florestal, em cada classe; $\mathrm{n}^{\circ} \mathrm{a}=$ número de árvores selecionadas para o corte.

\section{Cubagem e análise de tronco completa}

Depois da seleção, as árvores foram derrubadas para a realização da cubagem rigorosa de cada indivíduo pelo método de Smalian, sendo mesurados os diâmetros a altura de $0,1 \mathrm{~m}, 0,5 \mathrm{~m}, 0,9$ $\mathrm{m}, 1,30 \mathrm{~m}, 2,30$ e assim sucessivamente de $1 \mathrm{em} 1 \mathrm{~m}$ até o diâmetro mínimo de $8 \mathrm{~cm}$ (valor comercial estipulado pela empresa).

Após a cubagem dos indivíduos, procedeu-se a retirada de discos para análise de tronco completa (ANATRO). Seis discos foram coletados, nas alturas absolutas de 0,1 e 1,3 m também nas alturas relativas de $25 \%, 50 \%, 75 \%$ da altura total da árvore (h) e na altura comercial (diâmetro igual a $8 \mathrm{~cm}$ ). Os discos foram secos em estufa na temperatura de $60^{\circ} \mathrm{C}$ durante 48 horas. Após a secagem, foram submetidos ao polimento superficial com uma sequência crescente de lixas abrasivas de diferentes granulometrias $\left(60,120\right.$ e 320 grãos $\left./ \mathrm{mm}^{2}\right)$ para facilitar a visualização dos anéis de crescimento.

$\mathrm{Na}$ sequência, realizou-se a marcação dos anéis de crescimento. Para isso, quatro raios foram delimitados, nos quais marcações foram feitas evidenciando o término de cada anel de crescimento para facilitar a identificação no momento da medição.

Os discos foram digitalizados (resolução de 600 e 1200 dpi) e a espessura dos anéis de crescimento diamétrico foram medidas (sentido casca-medula) no aplicativo computacional de análise de imagem Image Pro Plus® versão 4.5, para a plataforma Windows.

A partir dos valores obtidos com a medição dos anéis de crescimento, determinou-se a média anual dos raios para cada indivíduo amostrado, possibilitando a criação de curvas de crescimento para cada árvore, demostrando as características de progressão do incremento anual da população para cada área.

\section{Modelagem do crescimento}

Com os dados processados oriundos da ANATRO fez-se a modelagem de crescimento das variáveis dendrométricas (diâmetro, altura e volume) para o grupo de árvores da Área 1 e da Área 2. O modelo utilizado foi o modelo de Chapman Richards sendo expresso pela fórmula (2).

$y=\beta 0 .\left(1-e^{-\beta 1 * x i}\right)^{\beta 2}$

Em que: $y=$ Variável dependente dap, altura, volume; $\mathrm{xi}=$ Variável independente (idade); $\beta 0, \beta 1$ e $\beta 2=$ Coeficientes.

Através modelo de Chapman - Richards, realizou-se a projeção do dap, altura e volume para as duas áreas amostrais (Área 1 e Área 2). Em ambas as áreas se aplicou a taxa de influência da resinagem no crescimento encontrada por Figueiredo Filho et al. (1992) sendo diâmetro $(14,49 \%)$, altura $(12,02$ $\%)$, volume $(14,99 \%)$, estabelecendo assim 4 cenários diferentes, ou seja, duas situações para cada área. Para o ajuste do modelo foi utilizado o algoritmo de Gauss Newton. As projeções foram realizadas levando em consideração o manejo utilizado atualmente pela empresa, no qual a resinagem inicia-se aos 12 anos de idade com duração de 8 anos.

\section{Afilamento}

Através dos dados da cubagem rigorosa das árvores pelo método de Smaliam, contendo diâmetro a altura do peito (DAP), altura total (ht), diâmetro em 
diferentes alturas (di) e altura ao longo do fuste (hi), ajustou-se o modelo de afilamento de Schöepfer (1996) descrito na fórmula (3) para o grupo de árvores das diferentes áreas e para o agrupamento das duas áreas, a fim de obter a estimativa de volume e diâmetro em qualquer secção do tronco.

$\frac{\mathrm{di}}{\mathrm{dap}}=\beta_{0}+\beta_{1}\left(\frac{\mathrm{hi}}{\mathrm{h}}\right)+\beta_{2}\left(\frac{\mathrm{hi}}{\mathrm{h}}\right)^{2}+\beta_{3}\left(\frac{\mathrm{hi}}{\mathrm{h}}\right)^{3}+\beta_{4}\left(\frac{\mathrm{hi}}{\mathrm{h}}\right)^{4}+\beta_{5}\left(\frac{\mathrm{hi}}{\mathrm{h}}\right)^{5}+\varepsilon \mathrm{i}$

Em que: dap: Diâmetro à altura do peito $(\mathrm{cm}) ;$ di $=$ diâmetro em diferentes alturas $(\mathrm{cm}) ; \mathrm{h}=$ altura total da árvore (m); hi = altura ao longo do fuste $(\mathrm{m}) ; \beta 0, \beta 1, \beta 2, \beta 3, \beta 4$ e $\beta 5=$ coeficientes; $\varepsilon \mathrm{i}=$ erro aleatório.

Após o ajuste das equações, os coeficientes obtidos foram empregados para obter os valores estimados de diâmetro e volume. Para obtenção do volume, foi aplicada a técnica de integração (fórmula 4), na qual a expressão propicia obter os volumes correspondentes a qualquer porção da árvore.

$\mathrm{V}=\int_{\mathrm{h} 2}^{\mathrm{h} 1} \frac{\pi}{40000} \mathrm{di}^{2} \delta \mathrm{h}$

Em que: $\mathrm{V}=$ volume total ou parcial estimado $\left(\mathrm{m}^{3}\right)$; $\mathrm{h} 1=$ limite inferior utilizado no processo de integração (m); h2 = limite superior usado no processo de integração $(\mathrm{m})$; di= diâmetro $(\mathrm{cm})$ medido às alturas hi, ao longo do fuste; $h=$ altura total (m).

\section{Análise econômica}

Para realização da avaliação econômica, determinou-se seis cenários. Como já informado anteriormente, procedeu-se para ambas as áreas (Área 1 e Área 2), a criação de cenários com árvores resinadas e não resinadas, conforme demostrado na Tabela 3.

Tabela 3. Caracterização dos diferentes cenários para simulação da análise econômica considerando ou não a resinagem.

\begin{tabular}{lcccccc}
\hline \multirow{2}{*}{ Características } & \multicolumn{7}{c}{ Cenários } \\
\cline { 2 - 7 } & \multicolumn{2}{c}{ Área 1 } & \multicolumn{5}{c}{ Área 2 } \\
\cline { 2 - 7 } & $\mathbf{1}(\mathbf{A})$ & $\mathbf{1}(\mathbf{B})$ & $\mathbf{2}(\mathbf{A})$ & $\mathbf{2}(\mathbf{B})$ & $\mathbf{2}(\mathbf{C})$ & $\mathbf{2}(\mathbf{D})$ \\
\hline Árvores/ha & 800 & 800 & 1.180 & 1.180 & 1.180 & 1.180 \\
Manejo & Não resinado & Resinado & Não resinado & Resinado & Resinado & Resinado \\
kg resina/árv. & ---- & 3,0 & ---- & 3,0 & 2,5 & 2,0 \\
\hline
\end{tabular}

Em que: ha = Hectares; kg= Quilograma; árv.= Árvore.

Atualmente a empresa trabalha com uma densidade de 800 árvores/ha para extração da resina, o que resulta em $3 \mathrm{~kg}$ árvore/ano. Por esse motivo, para a Área 2 (por desconhecer a quantidade de resina gerada para 1180 árvores/ha, sabendo-se que a densidade populacional possui interferência direta na quantidade de resina gerada) empregou-se cenários com distintas quantidades de resina produzida (2, 2,5 e $3 \mathrm{~kg}$ /árvore). Os dados referentes a produção, custos e receitas do sistema foram disponibilizados pela empresa proprietária da área. A partir dessas informações, foi possível formular o fluxo de caixa para os diferentes cenários avaliados.

A partir da elaboração do fluxo de caixa, determinou-se os índices de viabilidade econômica, utilizando uma Taxa Mínima de Atratividade (TMA) de $8 \%$. Os métodos mais indicados para esse tipo de análise no setor florestal são os que consideram o valor do capital no tempo, pois os investimentos têm retornos em longo prazo (Vitale e Miranda 2010). Assim, os métodos utilizados neste trabalho foram VPL, TIR e Razão B/C.

Calculou-se o VPL com base na equação 5, apresentada por Rezende e Oliveira (2008) como:

$$
\mathrm{VPL}=\sum_{\mathrm{j}=0}^{\mathrm{n}} \frac{(\mathrm{Rj})}{(1+\mathrm{i})^{\mathrm{j}}}-\sum_{\mathrm{j}=0}^{\mathrm{n}} \frac{(\mathrm{C} \mathrm{j})}{(1+\mathrm{i})^{\mathrm{j}}}
$$

Em que: $\mathrm{C} j=$ Custo no final do ano “ $\mathrm{j}$ ” ou do período considerado; $\mathrm{Rj}=$ Receita no final do ano “ $\mathrm{j}$ ” ou do período considerado; $\mathrm{i}$ = Taxa de desconto, expressa; $\mathrm{j}=$ Período em que a receita ou o custo ocorrem; $\mathrm{n}=$ duração do projeto, em anos, ou em número de períodos.

A TIR, por sua vez, teve como base a equação 6, também de acordo com Rezende e Oliveira (2008):

$$
\mathrm{TIR}=\sum_{\mathrm{j}=0}^{\mathrm{n}} \frac{(\mathrm{Rj})}{(1+\mathrm{TIR})^{\mathrm{j}}}-\sum_{\mathrm{j}=0}^{\mathrm{n}} \frac{(\mathrm{Cj})}{(1+\mathrm{TIR})^{\mathrm{j}}}=0
$$

Em que: $\mathrm{Rj}=$ Valor das receitas no ano $\mathrm{j} ; \mathrm{Cj}=$ Valor dos custos no ano j; i = Taxa de desconto anual, expressa em forma decimal; $\mathrm{j}=$ período em que a receita ou o custo ocorrem; $n=$ duração do projeto, em anos, ou em número de períodos.

A Razão Benefício Custo (Razão B/C) foi calculada conforme com Rezende e Oliveira (2008), por meio da seguinte equação: 
Razão B/C $=\frac{\sum_{j=0}^{n} R_{j} /(1+i)^{j}}{\sum_{j=0}^{n} C_{j} /(1+i)^{i}}$

Em que: $\mathrm{Rj}=$ Valor das receitas no ano $\mathrm{j} ; \mathrm{C}_{\mathrm{j}}=$ Valor dos custos no ano $\mathrm{j}$; $\mathrm{i}=$ Taxa de desconto anual, expressa em forma decimal; $\mathrm{j}=$ período em que a receita ou o custo ocorrem; $\mathrm{n}=$ duração do projeto, em anos, ou em número de períodos.

De acordo com Rezende e Oliveira (2008), pode-se dizer que, de forma geral, quando a Razão B/C $>1$, o VPL é maior que 0 e a TIR é maior que a TMA, sendo o projeto considerado economicamente viável.
Embora cada um dos métodos acima descrito possuam vantagens e desvantagens, todos eles são apropriados para auxiliar os administradores na tomada de decisão, com respeito às oportunidades de investimento no campo florestal (Berguer 1980). Os dados de produção e custos determinados para cada cenário são apresentados na Tabela 4.

As análises foram realizadas no Software Microsoft Excel e os gráficos no ambiente R ( $R$ Development Core Team, 2019) através do pacote ggplot2 (Wickham H, 2016).

Tabela 4. Valores da produção e custos para os diferentes cenários.

\begin{tabular}{lcccccc}
\hline \multicolumn{1}{c}{ Valores $(\mathbf{R} \$)$} & \multicolumn{6}{c}{ Cenários } \\
\cline { 2 - 7 } & $\mathbf{1 ~ ( A )}$ & $\mathbf{1 ~ ( B )}$ & $\mathbf{2 ( A )}$ & $\mathbf{2 ( B )}$ & $\mathbf{2}(\mathbf{C )}$ & $\mathbf{2}(\mathbf{D})$ \\
\hline Produção de breu/ha & --- & $6.554,68$ & --- & $9.668,16$ & $8.056,80$ & $6.445,44$ \\
Produção de terebintina/ha & --- & $2.467,92$ & --- & $3.640,18$ & $3.033,49$ & $2.426,79$ \\
Produção de madeira/ha & $45.887,89$ & $36.733,96$ & $71.698,99$ & $59.248,59$ & 59248,59 & $59.248,59$ \\
Custo de oport. da terra/ha & $9.518,40$ & $9.518,40$ & $9.518,40$ & $9.518,40$ & $9.518,40$ & $9.518,40$ \\
Custos da resinagem/ha & --- & $4.920,00$ & --- & $7.257,00$ & $6.047,50$ & $4.838,00$ \\
Custos da colheita/há & $17.971,13$ & $15.002,68$ & $27.614,45$ & $23.929,07$ & $23.929,07$ & $23.929,07$ \\
Custos adm. e silviculturais/ha & $2.540,98$ & $2.540,98$ & $2.540,98$ & $2.540,98$ & $2.540,98$ & $2.540,98$ \\
\hline
\end{tabular}

Em que: adm.= Administrativos; $1(\mathrm{~A})=$ Área 1 e árvores não resinadas; $1(\mathrm{~B})=$ Área 1, árvores resinadas com produção de 3kg/árvore; 2 (A)= Área 2 e árvores não resinadas; 2 (B)= Área 2, árvores resinadas com produção de 3kg/árvore; $(C)=$ Área 2, árvores resinadas com produção de 2,5kg/árvore; 2 (D)= Área 2, árvores resinadas com produção de $2 \mathrm{~kg}$ /árvore.

\section{Resultados e discussões}

Análise de tronco completa - ANATRO

Os indivíduos selecionados para cubagem e ANATRO foram escolhidos com base na distribuição diamétrica encontrada para cada situação provenientes dos dados do inventário florestal (Figura 2).

A distribuição da área 1 e 2 tem maior concentração de indivíduos nas classes de diâmetro centrais, fato esse muito comum em florestas plantadas, devido serem formadas por uma única espécie e equiâneas (Lima e Leão 2013).

$\mathrm{O}$ crescimento individual das 60 árvores amostradas é demostrado na Figura 3. Conferindo o crescimento anual das árvores amostradas com as características das áreas descritas na Tabela 1, identifica-se que, assim como estudos realizados por Flach (2007), os tratos silviculturais (roçada, raleio e desbaste) interferem diretamente no crescimento em diâmetro das árvores.

$\mathrm{Na}$ Área 1(Figura 3) nota-se que, após a aplicação da roçada e do desbaste nos anos de 2004, 2005 e 2010, que resultou na redução da densidade populacional, houve um aumento considerável no desenvolvimento dos indivíduos amostrados nos anos posteriores. O mesmo pode ser visto na Área 2, observando-se um pico no crescimento dos indivíduos desse local nos anos subsequentes à 2004 (ano que foi realizado as intervenções), confirmando a influência da densidade no crescimento em diâmetro. O incremento máximo foi de 2,18 e 3,28 $\mathrm{cm} \mathrm{ano}^{-1}$, respectivamente área 1 e 2 . As mesmas características também foram encontradas por Inoue et al. (2011) que estudaram a influência da densidade em um povoamento de Pinus taeda L., verificando que a redução da densidade populacional implicou num maior desenvolvimento das árvores em diâmetro, cerca de 2,3 vezes maiores em povoamento com maior densidade. Avaliando o crescimento do Pinus taeda L. em Santa Catarina, TOPANOTTI et al. (2021) também constataram que as árvores da referida espécie tiveram aumento no diâmetro nos anos seguinte após a realização de intervenção no talhão onde estavam situadas. O ganho em diâmetro pode influenciar no aumento das médias de volume, com a aumento de intensidade de desbastes (DOBNER JR, 2014) 


\section{Lima et al.}

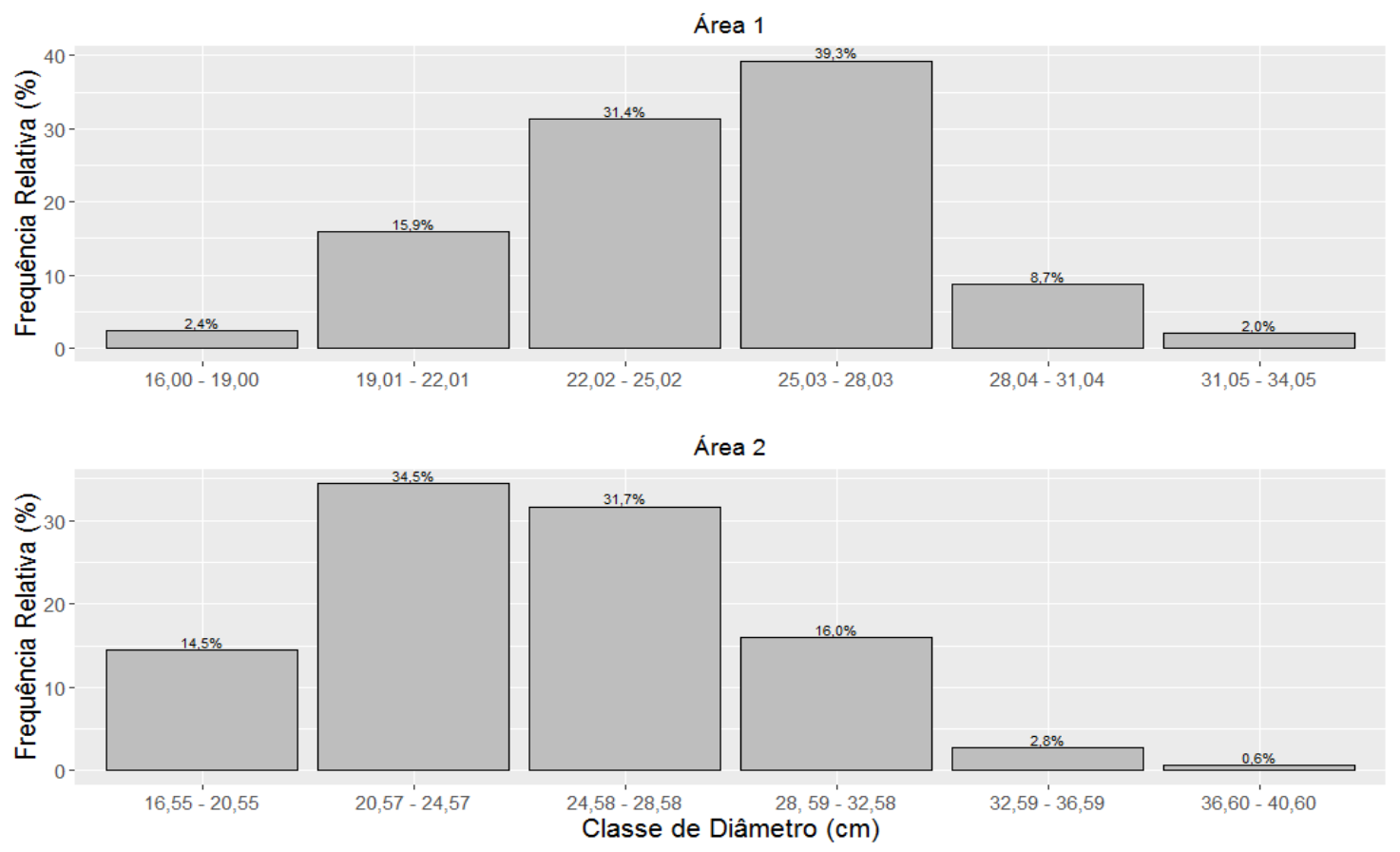

Figura 2. Distribuição diamétrica para as diferentes classes de diâmetro presentes para Área 1 e Área 2.

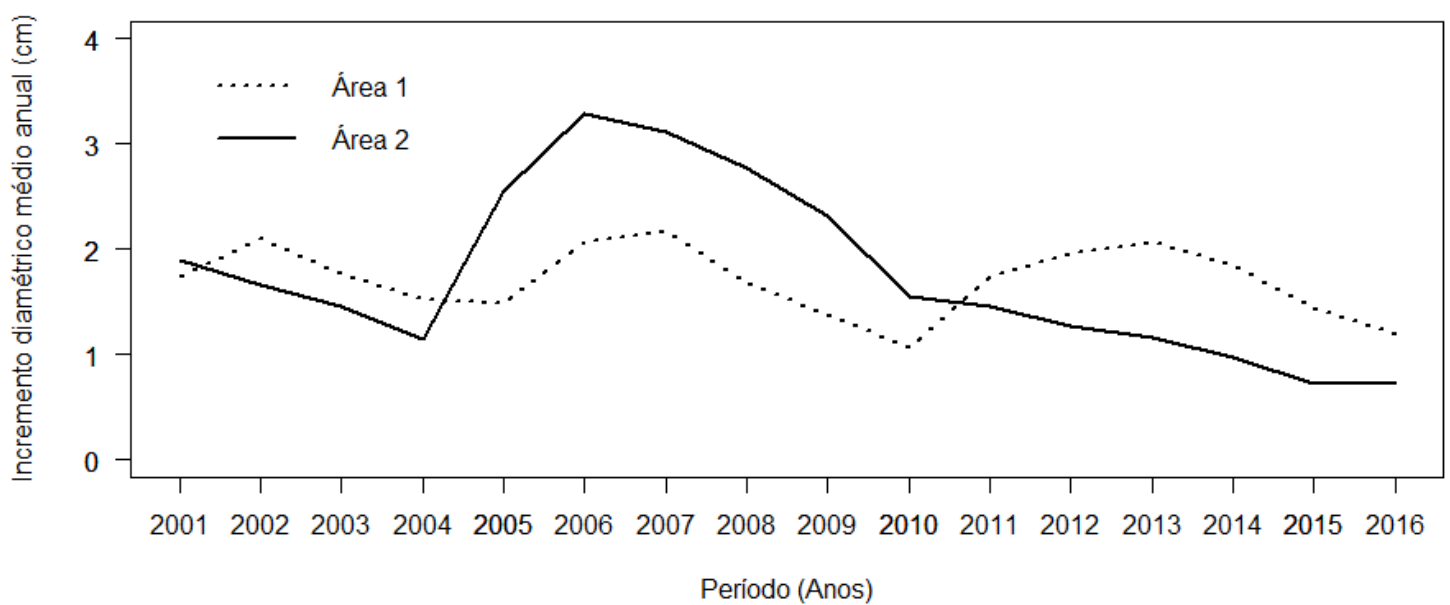

Figura 3. Incremento diamétrico médio anual $\left(\mathrm{cm} \mathrm{ano}^{-1}\right)$ das árvores amostradas para as diferentes áreas: Área $1 \mathrm{e}$ Área 2

\section{Curvas de crescimento}

Os valores da ANATRO de cada árvore são a base para a obtenção dos resultados a seguir. $\mathrm{Na}$ tabela 5 estão descritas as estatísticas do modelo Chapman - Richards que obteve as melhores estatísticas de avaliação de qualidade, tornando-o adequado para ao ajuste, para estimar o crescimento em função da idade para o grupo de árvores da Área
1 e da Área 2 para as seguintes variáveis: dap, altura e volume.

Foi aplicado para ambas as áreas, taxas de influência da resinagem encontradas por Figueiredo Filho et al (1992) no crescimento em diâmetro, altura e volume. A partir da seleção do melhor, determinou-se a projeção dessas variáveis para a Área 1 e Área 2 para a situação de árvores resinadas e árvores não resinadas (Figura 4). 


\section{Lima et al.}

Tabela 5. Estatística de ajuste do modelo de Chapman - Richards para Área 1 e 2.

\begin{tabular}{|c|c|c|c|c|c|c|c|c|}
\hline & \multicolumn{4}{|c|}{ Área 1} & \multicolumn{4}{|c|}{ Área 2} \\
\hline & \multicolumn{4}{|c|}{ Coeficientes } & & \multicolumn{3}{|c|}{ Coeficientes } \\
\hline & $\mathrm{S}_{\mathrm{yx}} \%$ & $\boldsymbol{\beta}_{0}$ & $\boldsymbol{\beta}_{1}$ & $\boldsymbol{\beta}_{2}$ & $\mathbf{S}_{\mathrm{yx}} \%$ & $\boldsymbol{\beta}_{0}$ & $\boldsymbol{\beta}_{1}$ & $\boldsymbol{\beta}_{2}$ \\
\hline dap & 21,7 & $53,42^{*}$ & $0,05^{*}$ & $1,23^{*}$ & 27,0 & $31,41^{*}$ & $0,17 *$ & $2,25^{*}$ \\
\hline $\begin{array}{c}\text { Altura } \\
\text { Volume }\end{array}$ & $\begin{array}{c}9,9 \\
56,2\end{array}$ & $\begin{array}{c}46,86^{*} \\
2,75^{*}\end{array}$ & $\begin{array}{l}0,04 * \\
0,07 *\end{array}$ & $\begin{array}{c}1,3^{*} \\
4,38^{*}\end{array}$ & $\begin{array}{l}10,4 \\
48,4\end{array}$ & $\begin{array}{c}49,35^{*} \\
2,5^{*}\end{array}$ & $\begin{array}{l}0,04 * \\
0,07 *\end{array}$ & $\begin{array}{l}1,09^{*} \\
3,45^{*}\end{array}$ \\
\hline
\end{tabular}

Syx $\%=$ erro padrão da estimativa; $\beta_{0}, \beta_{1}$ e $\beta_{2}=$ Coeficientes. * coeficiente significativo a $5 \%$ de probabilidade.
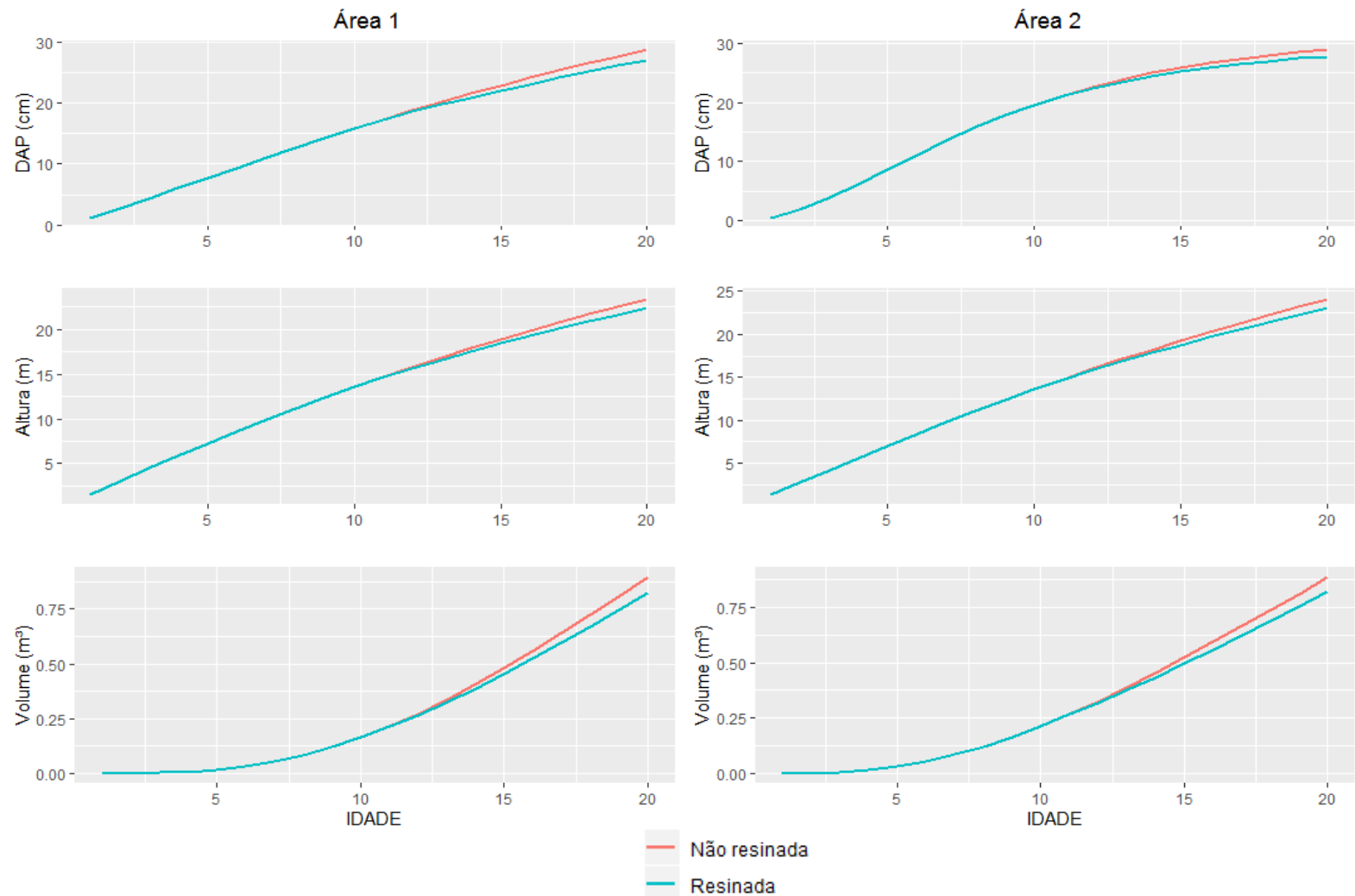

Figura 4. Simulação da projeção para o dap $(\mathrm{cm})$, altura $(\mathrm{m})$ e volume $\left(\mathrm{m}^{3}\right)$ em função da idade, para árvores resinadas e não resinadas para Área 1 e 2, ajustadas pelo modelo de crescimento Chapman-Richard.

Observa-se que as curvas de crescimento de todas as variáveis são semelhantes para as duas situações (Área 1 e 2) até o décimo primeiro ano. A partir dessa idade, é notável a influência no crescimento causado pelo início da técnica de resinagem para as variáveis apresentadas. Ribas et al. (1984) analisaram os efeitos da atividade de resinagem e concluíram que há diminuição o crescimento em DAP e altura de árvores resinadas. Isso condiz com os resultados do presente estudo onde se verificou mesmo comportamento.

Um fator importante a considerar na análise e comparação das curvas de desenvolvimento é o período de resinagem. A taxa de influência aplicada no trabalho resultou de um experimento de 4 anos de avaliação. Pesquisas realizadas nesse sentindo apontam que estes percentuais (taxas) tendem a aumentar com o ciclo de resinagem (Figueiredo Filho et al. 1992), levando-se a acreditar que as curvas de crescimento podem ainda tornarem-se mais distantes. Para Marcelino (2014) a perda de volume através da técnica de resinagem pode ser facilmente compensada pelo maior rendimento monetário e pela antecipação das receitas. Tratandose de pesquisas com mesma espécie e metodologias idênticas, para Luo et al. (1985) diferenças nos resultados de taxas de influência da resinagem no crescimento da madeira podem ser atribuídas ainda a diversos fatores, como ao sítio, concentração do ácido sulfúrico e ao comprimento das estrias.

Os resultados da Figura 4 mostram que as variáveis das árvores abordadas determinam o comportamento do rendimento da resina em Pinus elliottii. Trabalhando com a mesma espécie, mas na Tanzânia, o pesquisador Salim (2019) verificou que as variáveis DAP, altura, idade, espaçamento afetaram a produção de resina. Isso significa que para um melhor rendimento de resina, as relações intra e interárvores controladas pelo regime de manejo atual voltado para melhorar o volume de madeira do povoamento para a produção de são muito importantes. 
Afilamento

As estatísticas de ajuste e precisão demostraram que o modelo de afilamento de Schöepfer testado apresentou um alto coeficiente de determinação ajustado e um baixo erro padrão das estimativas. Considerando o dap para a área 1, obteve-se um $\mathrm{R}^{2}$ ajustado de 0,9821 enquanto para a área 2 esse valor foi 0,9857. Em relação ao Syx\% as áreas 1 e 2 registraram respectivamente $4,4 \%$ e $4,5 \%$.

A partir do modelo de Schöepfer e a da realização dos cálculos para obtenção das estimativas de diâmetro e volume em qualquer seção do tronco, estimou-se o volume por hectare presente em cada situação, assim como receita com a venda da madeira. Esses resultados são mostrados a seguir nas Tabelas 6, 7, 8 e 9 .

Tabela 6. Altura, diâmetro e volume em qualquer seção do tronco calculados por meio do modelo de Schöepfer para o cenário $1(\mathrm{~A})$.

\begin{tabular}{|c|c|c|c|c|c|c|c|c|c|}
\hline \multirow[b]{2}{*}{ Tora } & \multicolumn{2}{|c|}{ Altura (m) } & \multicolumn{2}{|c|}{ Diâmetro (cm) } & \multicolumn{4}{|c|}{ Volume $\left(\mathbf{m}^{3}\right)$} & \multirow{2}{*}{$\begin{array}{c}\text { Receita } \\
\text { R } \$ \text { ha } \\
\end{array}$} \\
\hline & Inf. & Sup. & Inf. & Sup. & Inf. & Sup. & $\mathrm{V}$ tora & V/ha & \\
\hline 1 & 0,1 & 2,8 & 33,14 & 26,99 & 0,0087 & 0,1938 & 0,1851 & 148,09 & $14.068,81$ \\
\hline 2 & 2,8 & 5,5 & 26,99 & 25,10 & 0,1938 & 0,3360 & 0,1421 & 113,70 & $8.527,79$ \\
\hline 3 & 5,5 & 8,2 & 25,10 & 23,83 & 0,3360 & 0,4633 & 0,1273 & 101,87 & $7.640,50$ \\
\hline 4 & 8,2 & 10,9 & 23,83 & 21,66 & 0,4633 & 0,5740 & 0,1107 & 88,54 & $6.640,80$ \\
\hline 5 & 10,9 & 13,6 & 21,66 & 18,45 & 0,5740 & 0,6600 & 0,0860 & 68,84 & $5.162,64$ \\
\hline 6 & 13,6 & 16,3 & 18,45 & 14,67 & 0,6600 & 0,7186 & 0,0586 & 46,86 & $2.422,81$ \\
\hline 7 & 16,3 & 19 & 14,67 & 10,67 & 0,7186 & 0,7531 & 0,0344 & 27,55 & $1.424,54$ \\
\hline 8 & 19 & 21,7 & 10,67 & 5,88 & 0,7531 & 0,7684 & 0,0154 & 12,28 & 0,00 \\
\hline
\end{tabular}

Em que= Inf.= Inferior; Sup.= Superior; V= Volume $\left(\mathrm{m}^{3}\right)$.

Tabela 7. - Altura, diâmetro e volume em qualquer seção do tronco calculados por meio do modelo de Schöepfer para o cenário $1(\mathrm{~B})$.

\begin{tabular}{cccccccccc} 
& \multicolumn{3}{c}{ Altura $(\mathbf{m})$} & \multicolumn{2}{c}{ Diâmetro $(\mathbf{c m})$} & \multicolumn{2}{c}{ Volume $\left(\mathbf{m}^{\mathbf{3}}\right)$} & \multicolumn{2}{c}{ Receita } \\
\cline { 2 - 10 } TORA & Inf. & Sup. & Inf. & Sup. & Inf. & Sup. & V tora & V/ha & R\$/ha \\
\hline 1 & 0,1 & 2,8 & 31,15 & 25,24 & 0,0077 & 0,1700 & 0,1623 & 129,85 & $9.738,77$ \\
2 & 2,8 & 5,5 & 25,24 & 23,50 & 0,1700 & 0,2945 & 0,1245 & 99,57 & $7.467,57$ \\
3 & 5,5 & 8,2 & 23,50 & 22,18 & 0,2945 & 0,4056 & 0,1112 & 88,92 & $6.669,31$ \\
4 & 8,2 & 10,9 & 22,18 & 19,86 & 0,4056 & 0,5003 & 0,0947 & 75,73 & $5.679,43$ \\
5 & 10,9 & 13,6 & 19,86 & 16,54 & 0,5003 & 0,5712 & 0,0709 & 56,72 & $4.254,06$ \\
6 & 13,6 & 16,3 & 16,54 & 12,75 & 0,5712 & 0,6171 & 0,0459 & 36,68 & $1.896,60$ \\
7 & 16,3 & 19,0 & 12,75 & 8,69 & 0,6171 & 0,6419 & 0,0249 & 19,89 & $1.028,22$ \\
8 & 19,0 & 21,7 & 8,69 & 3,28 & 0,6419 & 0,6506 & 0,0086 & 6,91 & 0,00 \\
\hline
\end{tabular}

Em que: Inf.= Inferior; Sup.= Superior; V= Volume $\left(\mathrm{m}^{3}\right)$.

Tabela 8. Altura, diâmetro e volume em qualquer seção do tronco calculados por meio do modelo de Schöepfer para o cenário $2(\mathrm{~A})$

\begin{tabular}{cccccccccc} 
& \multicolumn{3}{c}{ Altura $(\mathbf{m})$} & \multicolumn{3}{c}{ Diâmetro $(\mathbf{c m})$} & \multicolumn{3}{c}{ Volume $\left(\mathbf{m}^{\mathbf{3}}\right)$} \\
Tora & Inf. & Sup. & Inf. & Sup. & Inf. & Sup. & V tora & V/ha & R \$/ha \\
\hline 1 & 0,1 & 2,8 & 33,39 & 27,28 & 0,0089 & 0,1975 & 0,1887 & 222,66 & $21.152,57$ \\
2 & 2,8 & 5,5 & 27,28 & 25,35 & 0,1975 & 0,3426 & 0,1450 & 171,13 & $12.834,38$ \\
3 & 5,5 & 8,2 & 25,35 & 24,13 & 0,3426 & 0,4727 & 0,1301 & 153,52 & $11.514,15$ \\
4 & 8,2 & 10,9 & 24,13 & 22,12 & 0,4727 & 0,5871 & 0,1144 & 134,97 & $10.122,73$ \\
5 & 10,9 & 13,6 & 22,12 & 19,10 & 0,5871 & 0,6779 & 0,0909 & 107,24 & $8.042,78$ \\
6 & 13,6 & 16,3 & 19,10 & 15,46 & 0,6779 & 0,7417 & 0,0638 & 75,25 & $5.643,40$ \\
7 & 16,3 & 19 & 15,46 & 11,59 & 0,7417 & 0,7809 & 0,0392 & 46,21 & $2.388,99$ \\
8 & 19 & 21,7 & 11,59 & 7,20 & 0,7809 & 0,8003 & 0,0194 & 22,90 & 0,00 \\
\hline
\end{tabular}

Em que: Inf.= Inferior; Sup.= Superior; V= Volume $\left(\mathrm{m}^{3}\right)$. 
Tabela 9. Altura, diâmetro e volume em qualquer seção do tronco calculados por meio do modelo de Schöepfer para o cenário $2(\mathrm{~B})$.

\begin{tabular}{cccccccccc} 
& \multicolumn{3}{c}{ Altura (m) } & \multicolumn{2}{c}{ Diâmetro $(\mathbf{c m})$} & \multicolumn{2}{c}{ Volume $\left(\mathbf{m}^{\mathbf{3}}\right)$} & \multicolumn{2}{c}{ Receita } \\
\cline { 2 - 10 } TORA & Inf. & Sup. & Inf. & Sup. & Inf. & Sup. & V tora & V/ha & R\$/ha \\
\hline 1 & 0,1 & 2,8 & 32,04 & 26,04 & 0,0082 & 0,1806 & 0,1724 & 203,49 & $16.075,36$ \\
2 & 2,8 & 5,5 & 26,04 & 24,23 & 0,1806 & 0,3129 & 0,1323 & 156,15 & $11.711,14$ \\
3 & 5,5 & 8,2 & 24,23 & 22,95 & 0,3129 & 0,4314 & 0,1184 & 139,75 & $10.481,36$ \\
4 & 8,2 & 10,9 & 22,95 & 20,74 & 0,4314 & 0,5335 & 0,1022 & 120,54 & $90.40,71$ \\
5 & 10,9 & 13,6 & 20,74 & 17,51 & 0,5335 & 0,6118 & 0,0783 & 92,40 & $6.929,88$ \\
6 & 13,6 & 16,3 & 17,51 & 13,77 & 0,6118 & 0,6641 & 0,0523 & 61,69 & $3.189,45$ \\
7 & 16,3 & 19,0 & 13,77 & 9,79 & 0,6641 & 0,6939 & 0,0298 & 35,22 & $1.820,69$ \\
8 & 19,0 & 21,7 & 9,79 & 4,84 & 0,6939 & 0,7062 & 0,0122 & 14,45 & 0,00 \\
\hline
\end{tabular}

Em que= Inf.= Inferior; Sup.= Superior; V= Volume $\left(\mathrm{m}^{3}\right)$.

Análise econômica dos cenários gerados 10).

A partir dos dados de custos e receitas, elaborou-se um fluxo de caixa para cada cenário analisado (Tabela

Tabela 10. Valores por hectare dos custos e receitas referentes a cada cenário nas Áreas 1 e 2.

\begin{tabular}{cccccc}
\hline \multirow{2}{*}{ Área } & \multirow{2}{*}{ Cenários } & \multirow{2}{*}{ Situação } & \multicolumn{3}{c}{ Valores/ha } \\
\cline { 4 - 6 } & $\mathbf{1}(\mathbf{A})$ & $\mathbf{N R}$ & $15.015,26$ & $22.943,95$ & $7.928,69$ \\
$\mathbf{1}$ & $\mathbf{1}(\mathbf{B})$ & $\mathbf{R}$ & $33.211,03$ & $54.457,40$ & $21.246,37$ \\
& $\mathbf{2}(\mathbf{A})$ & $\mathbf{N R}$ & $19.836,91$ & $35.849,50$ & $16.012,58$ \\
& $\mathbf{2}(\mathbf{B})$ & $\mathbf{R}$ & $47.022,22$ & $82.857,67$ & $35.835,44$ \\
$\mathbf{2}$ & $\mathbf{2}(\mathbf{C})$ & $\mathbf{R}$ & $42.184,22$ & $73.985,44$ & $31.801,21$ \\
& $\mathbf{2}(\mathbf{D})$ & $\mathbf{R}$ & $37.346,22$ & $65.113,21$ & $27.766,98$ \\
\hline
\end{tabular}

Em que= FC:Fluxo de caixa (receita menos o custo); NR= Não resinado; $R=$ Resinado.

Tabela 11. Valores de VPL, Razão B/C e TIR para cada cenário avaliado.

\begin{tabular}{cccc}
\hline & \multicolumn{3}{c}{ Valores/ha } \\
\cline { 2 - 4 } Cenários & VPL $(\mathbf{R} \mathbf{\text { ) }}$ & R B/C & TIR (\%) \\
\hline $\mathbf{1}(\mathbf{A})$ & $-276,44$ & 0,97 & 7,6 \\
$\mathbf{1}(\mathbf{B})$ & $8.507,90$ & 1,39 & 17,9 \\
$\mathbf{2}(\mathbf{A})$ & $2.908,00$ & 1,23 & 11,0 \\
$\mathbf{2}(\mathbf{B})$ & $15.941,82$ & 1,54 & 21,4 \\
$\mathbf{2}(\mathbf{C})$ & $13.456,10$ & 1,51 & 19,9 \\
$\mathbf{2}(\mathbf{D})$ & $10.970,37$ & 1,46 & 18,2 \\
\hline
\end{tabular}

Em que: $\mathrm{VPL}=$ Valor presente líquido $(\mathrm{R} \$) ;$ Razão $\mathrm{B} / \mathrm{C}=$ Razão benefício/custo; TIR= Taxa interna de retorno (\%); ha=: hectare 
Como demostrado na Tabela 10, todos os cenários apresentaram fluxo de caixa positivo (receitas maiores que os custos) sendo observado o maior retorno para o cenário 2 (B) e o menor para 1 (A).

Analisando os cenários da Área 1, nota-se que o projeto em que a técnica de resinagem foi empregada teve um retorno superior de $\mathrm{R} \$ 13.317,68$ em relação ao não resinado, o que equivale a um acréscimo de $62,7 \%$.

Para a Área 2, os três cenários com resinagem apresentaram retornos superiores ao não resinado, com as seguintes valores e proporções: $\mathrm{R} \$ 19.822,86$ ou $55,3 \%$ para 2 (B), R $\$ 15.788,62$ ou $49,6 \%$ para 2 (C) e $\mathrm{R} \$ 11.754,40$ ou $42,3 \%$ para 2 (D).

A Tabela 11. apresenta a análise de viabilidade econômica (valores de VPL, Razão B/C e TIR), para os diferentes cenários.

Diante dos resultados exibidos pode-se verificar que o cenário 2 (B), em que se trabalhou com as características da Área 2 (1180 árvores/ha) e simulou o rendimento de $3 \mathrm{~kg}$ resina árvore/ano, apresentou os maiores valores de VPL (R\$ 15.941,82), Razão C/B (1,54) e TIR (21,4\%), demostrando ser o projeto mais atrativo economicamente. Os demais cenários considerados para a área 2, bem como o 1 (B) também são economicamente viáveis. Já o cenário 1 (A), que utilizou as características da Área 1 (800 árvores/ha) e não foi aplicado a resinagem demostrou-se inviável em todos os critérios utilizados.

No entanto, cabe destacar que o cenário considera uma situação hipotética, na qual uma área com maior densidade populacional produz a mesma quantidade de resina da área com menor número de árvores por hectare. Assim, tais resultados devem ser analisados com a devida atenção na confirmação da produção de goma resina, visando a tomada de decisão.

Estes resultados indicam que o alto valor de venda dos subprodutos da resinagem (breu e terebintina), neste estudo, fazem com que a produção de resina se torne mais rentável que a de madeira. Sendo assim, consegue-se afirmar que a resina produzida gera uma receita maior que a redução na produção de madeira.

Nota-se que mesmo com a aplicação da taxa de influência da resinagem no crescimento da madeira, os cenários em que a técnica de resinagem foi empregada, obtiveram os maiores valores de VPL, Razão B/C e TIR demostrando serem mais atrativos. Essa significativa elevação dos indicativos nos projetos com resinagem demonstra que a atividade representa uma excelente alternativa econômica para essas situações.

Os resultados encontrados nesta pesquisa corroboram com outros estudos, como o de Cordeiro e Lopes da Silva (2009), que comparou a rentabilidade de uma floresta de Pinus elliottii sob desbastes, para produção de madeira e produção de resina, constatando que projetos com a produção de resina tendem a ser mais rentáveis que os que trabalham somente com a produção de madeira.

Deve-se ressaltar que o mercado de comercialização da madeira e dos subprodutos da goma resina é instável e qualquer alteração nos mesmos pode modificar totalmente as receitas. Portanto, para utilização dos resultados aqui apresentados, como tomada de decisão, é necessário que se faça previamente uma atualização dos preços de vendas e dos custos.

Ainda na econômica da resina, Perez et al. (2013) avaliaram a importância e o potencial econômico desse produto em comunidades rurais na Espanha. Os autores mostraram a importância da resina na interrupção do declínio demográfico nas regiões onde esta atividade foi substancialmente desenvolvida. O trabalho também mostrou a relação entre a atividade de extração de resina e a evolução da população em municípios rurais. A exploração sustentável da resina pode contribuir para a manutenção e o desenvolvimento das áreas rurais comunidades, e a importância de ser usado como uma ferramenta para gerar empregos nas áreas rurais. Isso só demostra que a comercialização de resinagem não só influencia no aspecto social como também em maior rentabilidade financeira, pois assim destacamos o potencial que as florestas de Pinus teriam para a produção em toneladas, sendo possível aumentar fornecimento de resina para o mercado interno.

\section{Conclusão}

Os projetos em que a técnica de resinagem foi empregada para as diferentes áreas, obtiveram retornos superiores aos não resinados, com lucratividade superior de até $62,7 \%$. Nas duas áreas o processo de resinagem provocou uma redução nos valores das variáveis dendrométricas a partir do início desse processo em comparação com as árvores não resinadas. Porém, a resinagem indicou ser vantajosa mesmo que interfira diretamente no crescimento da árvore em diâmetro, volume e altura.

\section{Agradecimentos}

Os autores agradecem a Fundação de Amparo à Pesquisa e Inovação do Estado de Santa Catarina (FAPESC) ao laboratório de dendrometria da Universidade do Estado de Santa Catarina.

\section{Referências}

Alvares CA, Stape JL, Sentelhas PC, Gonçalves JLM, Sparovek G (2013) Köppen's climate classification map for Brazil. Meteorologische Zeitschrift, 22(6): 711-728.

ARESB - ASSOCIAÇÃO DOS RESINADORES DO BRASIL. Boletim Informativo Bimestral, São Paulo, n. diversos, 2017. 
Berguer R (1980) Análise benefício-custo: instrumento de auxílio para tomada de decisões na empresa florestal. IPEF - Circular Técnica, Piracicaba, n. 97.

Cordeiro SA, Lopes da Silva M (2009) Avaliação econômica de floresta de Pinus para produção de madeira e resina. Revista Agrogeoambiental, 1(2): 26-30.Costa Neto PLO (2002) Estatística. São Paulo: Edgar Blucher.

De Cademartori PHG, Gatto DA, Stangerlin DM, Schneid E, Hamm LG (2012) Qualidade da madeira serrada de Pinus elliottii Engelm. procedente de florestas resinadas. Cerne, 18(4): 577-583.

Dobner Jr. M (2014) Impacto do manejo de povoamentos na produtividade e qualidade da madeira de Pinus taeda. Tese (Doutorado em Ciências Florestais) - Universidade Federal do Paraná, UFPR, Curitiba, PR. 276p.

Ferreira JPRJ (2001) Análise da cadeia produtiva e estrutura de custos do setor brasileiro de produtos resinosos. Dissertação, Escola Superior de Agricultura "Luiz de Queiroz", Universidade de São Paulo. 120p.

Ferreira LMC (2014) Avaliação da influência da resinagem nas características da madeira. Estudo de alguns parâmetros da atividade resineira. Dissertação, Universidade de Tras-Os-Montes e Alto Douro. 81p.

Figueiredo Filho A, Machado SA, Hosokawa RT (1992) Avaliação econômica da resinagem em florestas de Pinus elliottii engelm. var. elliottii. IPEF - Circular Técnica, Piracicaba, n.45, p.48-63.

Flach FD (2007) Crescimento juvenil da regeneração espontânea de Pinus elliottii Eng. var. elliottii sob níveis de desbaste no litoral norte do Rio Grande do Sul. Dissertação, Universidade Federal do Rio Grande do Sul. 66p.

IBÁ - INDÚSTRIA BRASILEIRA DE ÁRVORES (2019) Relatório anual da IBÁ 2019, ano base 2018. Brasília: IBÁ.

Inoue MT, Figueiredo Filho A, Lima R (2011) Influência do espaço vital de crescimento na altura e diâmetro de Pinus taeda L. Scientia Forestalis, 39(91):337-385.

Garrido MAO, Poz R, Freitas JA, Rocha FT, Gurgel Garrido LMA (1998) Resinagem: Manual Técnico. Instituto Florestal - Secretaria do Meio Ambiente, $23 \mathrm{p}$.

Lima R, Inoue MT, Figueiredo Filho A, Araujo AJ, Macahado SA (2013) Efeito do Espaçamento no
Desenvolvimento Volumétrico de Pinus taeda L. Floresta e Ambiente, 20(2): 223-230.

Lima JPC, Leão JRA (2013) Dinâmica de crescimento e distribuição diamétrica de fragmentos de florestas nativa e plantada na Amazônia Sul Ocidental. Floresta e ambiente, 20(1): 70-79.

Marcelino FA (2004) Análise técnica e econômica da resinagem de Pinus elliottii Engelm. var. elliottii na região de Manduri, SP. Dissertação, Faculdade de Ciências Agronômicas, Universidade Estadual Paulista. 103p.

Neves GA, Martins CA, Mivasava J, Moura AF (2006) Aproveitamento do Pinus resinoso. Revista Madeira, 99.

Perez EA, Robredo FG, Tellez EA, Belda CF. Effects of the crisis in the resin sector on the demography of rural municipalities in Spain. Forest systems, v. 22, n. 1, p. 39-46, 2013.

R Development Core Team. 2019. R: A language and environment for statistical computing. $\mathrm{R}$ Foundation for Statistical Computing, Vienna, Austria.

Rezende JLP, Oliveira AD (2008) Análise Econômica e Social de Projetos Florestais. $2^{\circ}$ edição. Viçosa: UFV. 389p.

Ribas C; Gurgel Garrido LMA, Garrido MAO, Assini JL, Boas OV (1984) Produção de resina e influência no crescimento dendrométrico em árvores de Pinus elliottii Eng. var. elliottii, de diferentes diâmetros. Boletim Técnico. Instituto Florestal, São Paulo, 38(2): 155-163.

Rocha DP (2012) Evolução da resinagem de Pinus spp. no Brasil. Monografia, Universidade Federal Rural do Rio de Janeiro, Rio de Janeiro. 24p.

Rodrigues KCS (2008) Resina de Pinus no Sul do Brasil: Caracterização e Perspectivas. Revista da Madeira: 116.

Salim BS. Factors affecting pine resin productivity and its potential as source of revenue in the forest sector of Tanzania: a case study of Sao Hill Forest Plantation. 2019. Tese de Doutorado. Sokoine University of Agriculture.

Streck EV, Kámpf N, Dalmolin RD, Klamt E, Nascimento PC, Schneider P (2002) Solos do Rio Grande do Sul. Porto Alegre: EMATER/RS(UFRGS). 222p.

Topanotti LR, Vaz DR, Carvalho SDPC, TomazelloFilho M, Dobner Jr, M, Nicoletti MF (2021) Growth 
Lima et al.

and wood density of Pinus taeda L. as affected by shelterwood harvest in a two-aged stand in Southern Brazil. European Journal of Forest Research, p. $1-13,2021$.

Vitale V, Miranda GM (2010) Análise comparativa da viabilidade econômica de plantios de Pinus taeda e Eucalyptus dunnii na região centro-sul do Paraná. Floresta, 40(3): 469-476.

Wickham H (2016). ggplot2: Elegant Graphics for Data Analysis. Springer-Verlag New York. ISBN 978-3-319-24277-4, https://ggplot2.tidyverse.org. 